Article

\title{
Existence of Nonnegative Solutions of Linear Autonomous Functional Differential Equations
}

\section{Mihály Pituk}

Department of Mathematics, University of Pannonia, 8200 Veszprém, Hungary; pitukm@almos.uni-pannon.hu

Received: 19 June 2020; Accepted: 2 July 2020; Published: 5 July 2020

\begin{abstract}
It is shown that if we exclude the existence of nontrivial small solutions, then a linear autonomous functional differential equation has a nontrivial nonnegative solution if and only if it has a nonnegative eigenfunction.
\end{abstract}

Keywords: linear functional differential equation; nonnegative solution; small solution; characteristic equation; eigenfunction; decomposition theory

\section{Introduction}

Let $\mathbb{R}^{n}$ be the $n$-dimensional space of real column vectors with any norm $|\cdot|$. Given $r>0$, let $C=C\left([-r, 0], \mathbb{R}^{n}\right)$ denote the Banach space of continuous functions from $[-r, 0]$ into $\mathbb{R}^{n}$ with the supremum norm $\|\phi\|=\sup _{-r \leq \theta \leq 0}|\phi(\theta)|$ for $\phi \in C$.

Consider the linear autonomous retarded functional differential equation

$$
x^{\prime}(t)=L\left(x_{t}\right)
$$

where $L: C \rightarrow \mathbb{R}^{n}$ is a bounded linear functional and $x_{t} \in C$ is defined by $x_{t}(\theta)=x(t+\theta)$ for $\theta \in[-r, 0]$. According to the Riesz representation theorem, $L$ has the form

$$
L(\phi)=\int_{-r}^{0} d[\eta(\theta)] \phi(\theta), \quad \phi \in C,
$$

where $\eta:[-r, 0] \rightarrow \mathbb{R}^{n \times n}$ is a matrix function of bounded variation normalized such that $\eta$ is left continuous on $(-r, 0)$ and $\eta(0)=0$. Equation (1) includes as a special case the differential equation with a single delay

$$
x^{\prime}(t)=A x(t)+B x(t-r),
$$

where $A, B \in \mathbb{R}^{n \times n}$. By a solution of (1), we mean a function $x:[-r, \infty) \rightarrow \mathbb{R}^{n}$ which is continuous on $[-r, \infty)$, differentiable on $(0, \infty)$, and satisfies (1) for $t>0$. It is well-known [1] that, for every $\phi \in C$, Equation (1) has a unique solution with initial value $x_{0}=\phi$.

The characteristic equation of Equation (1) has the form

$$
\operatorname{det} \Delta(z)=0, \quad \Delta(z)=z I-\int_{-r}^{0} e^{z \theta} d \eta(\theta)
$$

where $z \in \mathbb{C}$ is a complex variable and $I$ is the $n \times n$ identity matrix.

A solution $x=\left(x_{1}, \ldots, x_{n}\right)^{T}:[-r, \infty) \rightarrow \mathbb{R}^{n}$ of (1) is called oscillatory if all coordinate functions $x_{1}, \ldots, x_{n}$ have arbitrarily large zeros. Otherwise, $x$ is called nonoscillatory. As usual, the superscript $T$ indicates the transpose. If Equation (4) has a real root $\mu \in \mathbb{R}$, then there exists $v \in \mathbb{R}^{n} \backslash\{0\}$ such that $\Delta(\mu) v=0$ and hence the corresponding eigensolution $x(t)=e^{\mu t} v$ is a nonoscillatory solution of (1). Thus, the existence of a real root of Equation (4) is sufficient for the existence of a nonoscillatory 
solution of (1). One of the key results in the oscillation theory of functional differential equations [2] states that the existence of a real root of Equation (4) is not only sufficient, but also necessary for the existence of a nonoscillatory solution of (1).

Theorem 1. ([3], Theorem 4.1) Equation (1) has a nonoscillatory solution if and only if Equation (4) has a real root.

The aim of this paper is to give a similar characterization for the existence of a nontrivial nonnegative solution of Equation (1). A solution $x=\left(x_{1}, \ldots, x_{n}\right)^{T}:[-r, \infty) \rightarrow \mathbb{R}^{n}$ of (1) is called nonnegative if all coordinate functions $x_{1}, \ldots, x_{n}$ are nonnegative on $[-r, \infty)$, or, equivalently, $x(t) \in \mathbb{R}_{+}^{n}$ for $t \geq-r$, where $\mathbb{R}_{+}^{n}$ is the nonnegative orthant, the set of those vectors in $\mathbb{R}^{n}$ which have nonnegative components. If there exist $\mu \in \mathbb{R}$ and $v \in \mathbb{R}_{+}^{n} \backslash\{0\}$ such that $\Delta(\mu) v=0$, then $x(t)=e^{\mu t} v$ is evidently a nontrivial nonnegative solution of Equation (1). Therefore it is natural to ask whether the existence of $\mu \in \mathbb{R}$ and $v \in \mathbb{R}_{+}^{n} \backslash\{0\}$ such that $\Delta(\mu) v=0$ is necessary for the existence of a nontrivial nonnegative solution of (1). The following simple example shows that the answer in general is negative.

Consider the two-dimensional system

$$
\begin{aligned}
& x_{1}^{\prime}(t)=x_{2}(t-1), \\
& x_{2}^{\prime}(t)=-x_{1}(t),
\end{aligned}
$$

a special case of Equation (3), where

$$
A=\left(\begin{array}{cc}
0 & 0 \\
-1 & 0
\end{array}\right), \quad B=\left(\begin{array}{ll}
0 & 1 \\
0 & 0
\end{array}\right)
$$

and $r=1$. The characteristic matrix is given by

$$
\Delta(z)=\left(\begin{array}{cc}
z & -e^{-z} \\
1 & z
\end{array}\right)
$$

In this case, there exist no $\mu \in \mathbb{R}$ and $v \in \mathbb{R}_{+}^{2} \backslash\{0\}$ such that $\Delta(\mu) v=0$. Otherwise, we obtain $0=\operatorname{det} \Delta(\mu)=\mu^{2}+e^{-\mu}>0$, a contradiction. On the other hand, Equation (5) has the nontrivial nonnegative solution $x=\left(x_{1}, x_{2}\right)^{T}:[-1, \infty) \rightarrow \mathbb{R}^{2}$ given by

$$
x_{1}(t)=\left\{\begin{array}{ll}
t^{2} & \text { for } t \in[-1,0] \\
0 & \text { for } t \in(0, \infty)
\end{array} \quad \text { and } \quad x_{2}(t)=0 \quad \text { for } t \in[-1, \infty) .\right.
$$

Note that the above nontrivial nonnegative solution $x$ is a small solution in the sense of the following definition [1]. A solution $x$ of Equation (1) is called a small solution if

$$
\lim _{t \rightarrow \infty} x(t) e^{k t}=0 \quad \text { for every } k \in \mathbb{R} .
$$

The zero solution is always a small solution. The question is whether there exist initial conditions $\phi \neq 0$ which generate small solutions. Such solutions are called nontrivial small solutions. A linear autonomous ordinary differential equation cannot have a nontrivial small solution. The existence of nontrivial small solutions of Equation (1) is a consequence of the fact that the phase space $C$ is infinite dimensional. As shown in ([1], Chap. 7, Corollary 8.1), Equation (1) has no nontrivial small solutions if and only if the exponential type of the characteristic function det $\Delta$ is equal to $n r$, or, equivalently, the system of eigenfunctions and generalized eigenfunctions of the generator of 
Equation (1) is complete. As a corollary, we have that Equation (3) has no nontrivial small solutions if and only if $\operatorname{det} B \neq 0$.

In this paper, we will show that if we exclude the existence of nontrivial small solutions, then the "natural" sufficient condition $\Delta(\mu) v=0$ for some $\mu \in \mathbb{R}$ and $v \in \mathbb{R}_{+}^{n} \backslash\{0\}$ is necessary for the existence of a nontrivial nonnegative solution of Equation (1).

The main result and its proof are given in Section 2. In Section 3, we briefly mention some results which are relevant to our study.

\section{Main Result}

Our main result is the following theorem.

Theorem 2. Suppose that Equation (1) has no nontrivial small solutions. Then Equation (1) has a nontrivial nonnegative solution if and only if there exist $\mu \in \mathbb{R}$ and $v \in \mathbb{R}_{+}^{n} \backslash\{0\}$ such that $\Delta(\mu) v=0$.

Before we present the proof of Theorem 2, we recall some facts from the decomposition theory of linear autonomous functional differential equations given in ([1], Chap. 7) and we establish two preliminary results.

It is known that Equation (1) generates in $C$ a strongly continuous semigroup $(T(t))_{t \geq 0}$, where $T(t): C \rightarrow C$ is a bounded linear operator, the so-called solution operator, defined by $T(t) \phi=x_{t}(\phi)$ for $t \geq 0$ and $\phi \in C, x_{t}(\phi)$ being the unique solution of (1) with initial value $x_{0}=\phi$. The infinitesimal generator $A: \mathcal{D}(A) \rightarrow C$ of this semigroup is defined by

$$
A \phi=\lim _{t \rightarrow 0+} \frac{1}{t}[T(t) \phi-\phi]
$$

whenever the limit exists in $C$. It is known that

$$
\mathcal{D}(A)=\left\{\phi \in C \mid \phi^{\prime} \in C, \phi^{\prime}(0)=L(\phi)\right\} \quad \text { and } \quad A \phi=\phi^{\prime} .
$$

The spectrum $\sigma(A)$ of the linear operator $A: \mathcal{D}(A) \rightarrow C$ is a point spectrum and it consists of the roots of Equation (4). In each strip $\alpha \leq|z| \leq \beta$, where $-\infty<\alpha<\beta<\infty$, Equation (4) has only a finite number of roots. Furthermore, if $\Lambda$ is a finite set of characteristic roots, then $C$ is decomposed by $\Lambda$ into a direct sum

$$
C=P_{\Lambda} \oplus Q_{\Lambda}
$$

where $P_{\Lambda}$ is the (realified) generalized eigenspace of $A$ associated with $\Lambda$ and $Q_{\Lambda}$ is the complementary subspace of $C$ such that $T(t) Q_{\Lambda} \subset Q_{\Lambda}$ for $t \geq 0$. Thus, each $\phi \in C$ can be written uniquely as

$$
\phi=\phi^{P_{\Lambda}}+\phi^{Q_{\Lambda}}, \quad \text { where } \phi^{P_{\Lambda}} \in P_{\Lambda} \text { and } \phi^{Q_{\Lambda}} \in Q_{\Lambda} .
$$

From now on, let $C_{+}$denote the set of nonnegative functions in $C$, i.e.,

$$
C_{+}=\left\{\phi \in C \mid \phi(\theta) \in \mathbb{R}_{+}^{n} \text { for all } \theta \in[-r, 0]\right\} .
$$

The following lemma will play an important role in the proof of Theorem 2.

Lemma 1. Suppose that Equation (1) has no nontrivial small solutions. If $x$ is a nontrivial nonnegative solution of Equation (1), then its Lyapunov exponent $\mu$ given by

$$
\mu=\mu(x)=\limsup _{t \rightarrow \infty} \frac{\ln \left\|x_{t}\right\|}{t}
$$


is finite, and if we let

$$
\Lambda_{0}=\{z \in \mathbb{C} \mid \operatorname{det} \Delta(z)=0, \operatorname{Re} z=\mu\},
$$

then there exists $\psi \in P_{\Lambda_{0}} \cap C_{+}$such that

$$
\|\psi\|=1 \quad \text { and } \quad x_{t}(\psi)=T(t) \psi \in P_{\Lambda_{0}} \cap C_{+} \text {for all } t \geq 0,
$$

where $P_{\Lambda_{0}}$ is the generalized eigenspace of the infinitesimal generator $A$ associated with $\Lambda_{0}$.

Proof. Let $x=x(\phi)$ be a nontrivial nonnegative solution of Equation (1) with initial value $x_{0}=\phi \in C_{+}$ and Lyapunov exponent $\mu=\mu(x)$. Then $x_{t}(\phi)=T(t) \phi \in C_{+}$for all $t \geq 0$. By the assumptions, $x$ cannot be a small solution and hence $\mu$ is finite (see [1], Chap. 7, Theorem 6.1). Define

$$
\Lambda=\{z \in \mathbb{C} \mid \operatorname{det} \Delta(z)=0, \operatorname{Re} z \geq \mu\}
$$

so that $C=P_{\Lambda} \oplus Q_{\Lambda}$. The generalized eigenspace $P_{\Lambda}$ can be further decomposed into the direct sum $P_{\Lambda}=P_{\Lambda_{0}} \oplus P_{\Lambda_{1}}$ with $\Lambda_{0}$ as in (11) and

$$
\Lambda_{1}=\{z \in \mathbb{C} \mid \operatorname{det} \Delta(z)=0, \operatorname{Re} z>\mu\} .
$$

Thus, writing $Q=Q_{\Lambda}, P=P_{\Lambda}, P_{0}=P_{\Lambda_{0}}$ and $P_{1}=P_{\Lambda_{1}}$ for brevity, we have that $C=P_{0} \oplus P_{1} \oplus Q$ and hence

$$
x_{t}(\phi)=x_{t}^{P_{0}}(\phi)+x_{t}^{P_{1}}(\phi)+x_{t}^{Q}(\phi)=T(t) \phi^{P_{0}}+T(t) \phi^{P_{1}}+T(t) \phi^{Q} \quad \text { for } t \geq 0,
$$

where $\phi^{P_{0}} \in P_{0}, \phi^{P_{1}} \in P_{1}$ and $\phi^{Q} \in Q$. All three subspaces $P_{0}, P_{1}$ and $Q$ are invariant under the solution semigroup. The generalized eigenspaces $P_{0}$ and $P_{1}$ are finite-dimensional and the solutions starting from $P_{0}$ and $P_{1}$ can be extended backward to all $t<0$. As a consequence, on $P_{0}$ and $P_{1}$ the solution semigroup can be extended to a group. It is known that for every $\epsilon>0$ there exists $K=K(\epsilon)>0$ such that the following exponential estimates hold:

$$
\begin{aligned}
\left\|T(t) \phi^{Q}\right\| & \leq K e^{(\mu-\epsilon) t}\left\|\phi^{Q}\right\|, \quad t \geq 0, \\
\left\|T(t) \phi^{P_{0}}\right\| & \leq K(1+|t|)^{m-1} e^{\mu t}\left\|\phi^{P_{0}}\right\|, \quad t \in(-\infty, \infty), \\
\left\|T(t) \phi^{P_{1}}\right\| & \leq K e^{(\mu+\epsilon) t}\left\|\phi^{P_{1}}\right\|, \quad t \leq 0,
\end{aligned}
$$

where $m$ is the maximum of the ascents of the characteristic roots from $\Lambda_{0}$ (see ([1], Sec. 7.6) and ([4], Equations (3.16) and (3.17))). Replacing $\phi^{P_{1}}$ with $T(t) \phi^{P_{1}}, t \geq 0$, in the last inequality and using the group property $T(-t) T(t)=T(0)=I$ on $P_{1}$, we find that

$$
\left\|T(t) \phi^{P_{1}}\right\| \geq K^{-1} e^{(\mu+\epsilon) t}\left\|\phi^{P_{1}}\right\|, \quad t \geq 0 .
$$

This implies that $\phi^{P_{1}}=0$. Otherwise, the last inequality, combined with (15) and the previous exponential estimates, would imply that $\mu=\mu(x) \geq \mu+\epsilon$, a contradiction. We claim that $\phi^{P_{0}} \neq 0$. Otherwise, by virtue of (15), we have that $x_{t}(\phi)=T(t) \phi^{Q}$ for $t \geq 0$, which, together with the exponential estimate on $Q$, implies that $\mu=\mu(x) \leq \mu-\epsilon$, a contradiction. From the exponential estimate on $P_{0}$, we find that

$$
\left\|T(t) \phi^{P_{0}}\right\| \geq K^{-1}(1+t)^{1-m} e^{\mu t}\left\|\phi^{P_{0}}\right\|, \quad t \geq 0 .
$$

Since $\phi^{P_{0}} \neq 0$, this, together with the exponential estimate on $Q$, implies that

$$
\frac{\left\|x_{t}^{Q}(\phi)\right\|}{\left\|x_{t}^{P_{0}}(\phi)\right\|}=\frac{\left\|T(t) \phi^{Q}\right\|}{\left\|T(t) \phi^{P_{0}}\right\|} \longrightarrow 0 \quad \text { as } t \rightarrow \infty .
$$


Hence

$$
\frac{\left\|x_{t}(\phi)\right\|}{\left\|x_{t}^{P_{0}}(\phi)\right\|}=\frac{\left\|x_{t}^{P_{0}}(\phi)+x_{t}^{Q}(\phi)\right\|}{\left\|x_{t}^{P_{0}}\right\|} \longrightarrow 1 \quad \text { as } t \rightarrow \infty
$$

and

$$
\frac{\left\|x_{t}^{Q}(\phi)\right\|}{\left\|x_{t}(\phi)\right\|} \longrightarrow 0 \quad \text { as } t \rightarrow \infty
$$

In particular,

$$
\frac{\left\|x_{n}^{P_{0}}(\phi)\right\|}{\left\|x_{n}(\phi)\right\|} \longrightarrow 1 \quad \text { as } n \rightarrow \infty
$$

Thus, $\left\{\left\|x_{n}(\phi)\right\|^{-1} x_{n}^{P_{0}}(\phi)\right\}_{n=1}^{\infty}$ is a bounded sequence in the finite-dimensional and hence closed subspace $P_{0}$ of $C$. Therefore there exist $\psi \in P_{0}$ with $\|\psi\|=1$ and a sequence $n_{k} \rightarrow \infty$ such that

$$
\frac{x_{n_{k}}^{P_{0}}(\phi)}{\left\|x_{n_{k}}(\phi)\right\|} \longrightarrow \psi \quad \text { as } k \rightarrow \infty
$$

It remains to show that $\psi$ has the desired properties. Since $\psi \in P_{0}$ and $P_{0}$ is invariant under the solution semigroup, we have that $x_{t}(\psi)=T(t) \psi \in P_{0}$ for $t \geq 0$. As shown before, $x_{t}^{Q}(\phi)=o\left(\left\|x_{t}(\phi)\right\|\right)$ as $t \rightarrow \infty$. Hence

$$
\frac{x_{n_{k}}(\phi)}{\left\|x_{n_{k}}(\phi)\right\|}=\frac{x_{n_{k}}^{P_{0}}(\phi)}{\left\|x_{n_{k}}(\phi)\right\|}+\frac{x_{n_{k}}^{\mathrm{Q}}(\phi)}{\left\|x_{n_{k}}(\phi)\right\|} \longrightarrow \psi \quad \text { as } k \rightarrow \infty .
$$

From this, using the nonnegativity of $x(\phi)$, the continuity and the semigroup property of $T(t)$, we find that

$$
T(t) \psi=\lim _{k \rightarrow \infty} \frac{T(t) x_{n_{k}}(\phi)}{\left\|x_{n_{k}}(\phi)\right\|}=\lim _{k \rightarrow \infty} \frac{T(t) T\left(n_{k}\right)(\phi)}{\left\|x_{n_{k}}(\phi)\right\|}=\lim _{k \rightarrow \infty} \frac{T\left(t+n_{k}\right)(\phi)}{\left\|x_{n_{k}}(\phi)\right\|}=\lim _{k \rightarrow \infty} \frac{x_{t+n_{k}}(\phi)}{\left\|x_{n_{k}}(\phi)\right\|} \in C_{+}
$$

for all $t \geq 0$.

Let $X$ be a real Banach space. A subset $K \subset X$ is called a cone if the following three conditions hold:

(i) $K$ is a nonempty, convex and closed subset of $X$,

(ii) $t K \subset K$ for all $t \geq 0$, where $t K=\{t x \mid x \in K\}$,

(iii) $K \cap(-K)=\{0\}$, where $-K=\{-x \mid x \in K\}$.

In the proof of Theorem 2, we will need the following result which gives a necessary and sufficient condition for the existence of a nontrivial orbit of a linear invertible map $M: X \rightarrow X$ which lies in a given cone $K$. By an orbit starting from $x \in X$, we mean the sequence of iterates $\left\{M^{m}(x)\right\}_{m=0}^{\infty}$. As usual, $M^{0}=I$, the identity on $X$.

Lemma 2. Let $K$ be a cone in a finite-dimensional real Banach space $X$. Suppose that $M: X \rightarrow X$ is a linear invertible operator. Then $M$ has an orbit belonging to $K \backslash\{0\}$ if and only if $M$ has a positive eigenvalue with an eigenvector in $\mathrm{K}$.

In the special case $X=\mathbb{R}^{n}$, Lemma 2 was proved in ([5], Theorem 3). Here we give a different argument which is valid in general finite-dimensional Banach spaces.

Proof of Lemma 2. If $v \in K$ is an eigenvector of $M$ corresponding to a positive eigenvalue $\rho$, then $M^{m}(v)=\rho^{m} v \in K \backslash\{0\}$. Thus, the orbit starting from $v$ belongs to $K \backslash\{0\}$. 
Now suppose that $M$ has an orbit starting from $y \in X$ which lies in $K \backslash\{0\}$. In particular, $y=M^{0}(y) \in K \backslash\{0\}$. Without loss of generality, we may (and do) assume that $\|y\|=1$. Otherwise, we replace $y$ with $\|y\|^{-1} y$ and use the cone property (ii). Let

$$
S=\left\{x \in K \mid\|x\| \leq 1 \text { and } M^{m}(x) \in K \text { for all } m=0,1, \ldots\right\}
$$

Evidently, $S$ is a convex closed subset of $X$ and $y \in S$. Define an operator $F: S \rightarrow X$ by

$$
F(x)=\frac{(1-\|x\|) y+\|x\| M(x)}{\|(1-\|x\|) y+\| x\|M(x)\|} \quad \text { for } x \in S \text {. }
$$

The cone property (iii), the fact that $y \neq 0$ and the invertibility of $M$ imply that $F$ is well-defined. Evidently, $F$ is continuous on $S$. The definition of $S$ and the cone properties (i) and (ii) imply that $F(S) \subset S$. By Brouwer's fixed point theorem, there exists $v \in S$ such that $F(v)=v$. Since $\|v\|=\|F(v)\|=1$, it follows that

$$
v=F(v)=\frac{M(v)}{\|M(v)\|} .
$$

Hence $M(v)=\rho v$, where $\rho=\|M(v)\|$. Since $v \neq 0$ and $M$ is invertible, we have that $M(v) \neq 0$ and hence $\rho=\|M(v)\|>0$. Thus, $\rho$ is a positive eigenvalue of $M$ and $v \in K$ is a corresponding eigenvector.

Now we can give a proof of Theorem 2 which follows similar lines as the proof of a Perron type theorem for positive solutions of a perturbed system of nonautonomous linear functional differential equations in [6].

Proof of Theorem 2. As noted before, if there exist $\mu \in \mathbb{R}$ and $v \in \mathbb{R}_{+}^{n} \backslash\{0\}$ such that $\Delta(\mu) v=0$, then $x(t)=e^{\mu t} v$ is a nontrivial nonnegative solution of (1).

Now suppose that Equation (1) has a nontrivial nonnegative solution $x$. By Lemma 1, the Lyapunov exponent $\mu=\mu(x)$ is finite. Let $\Lambda_{0}$ be the spectral set defined by (11). As noted before, the associated generalized eigenspace $P_{0}=P_{\Lambda_{0}}$ of (1) is finite-dimensional and invariant under the solution semigroup $(T(t))_{t \geq 0}$ with infinitesimal generator $A$ given by (7). Since $P_{0} \subset \mathcal{D}(A)$ is finite-dimensional, it is a closed subspace of $C$ and therefore we can define the subspace semigroup $\left(T_{0}(t)\right)_{t>0}$ on $P_{0}$ by $T_{0}(t)=\left.T(t)\right|_{P_{0}}$, the restriction of $T(t)$ to $P_{0}$ ([7], Paragraph I.5.12). Its generator is $A_{0}=\left.A\right|_{P_{0}}$ with domain $\mathcal{D}\left(A_{0}\right)=P_{0}$ ([7], Paragraph II.2.3) and $\sigma\left(A_{0}\right)=\Lambda_{0}$. Since $\operatorname{dim} P_{0}<\infty$, the generator $A_{0}: P_{0} \rightarrow P_{0}$ is bounded and therefore $T_{0}(t)=e^{t A_{0}}$ for $t \geq 0$ ([8], Chap. I, Sec. 1.1). According to the spectral mapping theorem [8], we have that

$$
\sigma\left(T_{0}(t)\right)=e^{t \sigma\left(A_{0}\right)}=\left\{e^{z t} \mid z \in \Lambda_{0}\right\}, \quad t \geq 0
$$

Define $K=P_{0} \cap C_{+}$. By Lemma 1, there exists $\psi \in K \backslash\{0\}$ such that $T_{0}(t) \psi \in K$ for all $t \geq 0$. Since $0 \notin \sigma\left(T_{0}(t)\right)$, we have that $T_{0}(t): P_{0} \rightarrow P_{0}$ is invertible for $t \geq 0$. Hence

$$
T_{0}(t) \psi \in K \backslash\{0\}, \quad t \geq 0
$$

Evidently, $K$ is a cone in $P_{0}$. Let $\left\{t_{k}\right\}_{k=1}^{\infty}$ be a sequence of positive numbers such that $t_{k} \rightarrow 0$ as $k \rightarrow \infty$. For every fixed $k$, consider the linear operator $M=T_{0}\left(t_{k}\right)$ in $P_{0}$. By the semigroup property, we have that $M^{m}=T_{0}\left(m t_{k}\right)$ for $m=0,1,2, \ldots$ This, together with (20), implies that $M$ has an orbit which belongs to $K \backslash\{0\}$. By the application of Lemma 2, we conclude that $M=T_{0}\left(t_{k}\right)$ has a positive eigenvalue $\rho_{k}$ with an eigenvector $\psi_{k} \in K$. Without loss of generality, we may assume that $\left\|\psi_{k}\right\|=1$. 
Otherwise, we replace $\psi_{k}$ with $\left\|\psi_{k}\right\|^{-1} \psi_{k}$ which belongs to $K$ by the cone property (ii). By virtue of (19), $\rho_{k}=e^{z t_{k}}$ for some characteristic root $z$ with $\operatorname{Re} z=\mu$. From this, using the positivity of $\rho_{k}$, we find that

$$
\rho_{k}=\left|\rho_{k}\right|=\left|e^{z t_{k}}\right|=e^{t_{k} \operatorname{Re} z}=e^{\mu t_{k}}
$$

Hence

$$
T_{0}\left(t_{k}\right) \psi_{k}=e^{\mu t_{k}} \psi_{k}, \quad \psi_{k} \in K, \quad\left\|\psi_{k}\right\|=1
$$

for $k=1,2, \ldots$. Since $\left\{\psi_{k}\right\}_{k=1}^{\infty}$ is a bounded sequence in the finite-dimension Banach space $P_{0}$, there exists a subsequence $\left\{\psi_{k_{j}}\right\}_{j=1}^{\infty}$ of $\left\{\psi_{k}\right\}_{k=1}^{\infty}$ such that the limit

$$
\chi=\lim _{j \rightarrow \infty} \psi_{k_{j}}
$$

exists in $P_{0}$. Evidently, $\|\chi\|=1$. Since $K$ is closed subset of $P_{0}$, we have that $\chi \in K$. From (21), we find that

$$
\frac{T_{0}\left(t_{k_{j}}\right) \psi_{k_{j}}-\psi_{k_{j}}}{t_{k_{j}}}=\frac{e^{\mu t_{k_{j}}}-1}{t_{k_{j}}} \psi_{k_{j}}
$$

for $j=1,2, \ldots$ From this, letting $j \rightarrow \infty$, using (22) and the fact that

$$
\left.\frac{d^{+}}{d t}\right|_{t=0} T_{0}(t)=\left.\frac{d^{+}}{d t}\right|_{t=0} e^{t A_{0}}=A_{0},
$$

we obtain

$$
\chi^{\prime}=A_{0} \chi=\mu \chi
$$

Hence $\chi(\theta)=\chi(0) e^{\mu \theta}$ for $\theta \in[-r, 0]$. Since $0 \neq \chi \in K \subset C_{+}$, we have that $v:=\chi(0) \in \mathbb{R}_{+}^{n} \backslash\{0\}$. Finally, $\chi \in P_{0} \subset \mathcal{D}(A)$ implies that $\chi^{\prime}(0)=L(\chi)$ which is equivalent to $\Delta(\mu) v=0$.

\section{Discussion}

The basic oscillation theorem for differential equations with constant coefficients and several delays was obtained by Arino and Györi [9]. A generalization to a class of linear differential equations with distributed delays was given by Győri and Krisztin [3]. Krisztin [10] showed that linear functional differential equations of mixed type may have nonoscillatory solutions in spite of the nonexistence of a real root of the characteristic equation. Henry [11] proved that small solutions of linear autonomous retarded functional differential equations must vanish after some time. Henry's theorem was improved by Verduyn Lunel [12]. Further information on small solutions and the completness of the eigenfunctions and generalized eigenfunctions of linear autonomous functional differential equations can be found in the monographs by Hale and Verduyn Lunel [1] and Dieckman et al. [13]. Small solutions for nonlinear equations were studied for the first time by Mallet Paret [14], who showed that they do not exist on the attractor of certain nonlinear scalar delay differential equations. For later results, see the papers by Cao [15], Arino [16], Cooke and Verduyn Lunel [17], Mallet Paret and Sell [18], Braverman et al. [19], Garab [20] and the references therein.

The main result of this paper, Theorem 2, is closely related to Theorem 1.5 from our recent work [6], which shows that if $K$ is a cone in $\mathbb{R}^{n}$, then Equation (1) has a positive solution with respect to the partial order induced by $K$ if and only if Equation (4) has a real root with a positive eigenfunction. In the case $K=\mathbb{R}_{+}^{n}$, Theorem 2 improves ([6], Theorem 1.5) in the sense that while the latter theorem applies only to positive solutions, Theorem 2 provides a similar conclusion for the larger class of nontrivial nonnegative solutions. It should be noted that ([6], Theorem 1.5) is a corollary of a more general Perron type theorem for positive solutions of a perturbed system of functional differential equation with a long proof. We believe that the above short proof of Theorem 2 can be of interest. 
Funding: This research was funded by the Hungarian National Research, Development and Innovation Office grant no. K120186 and Széchenyi 2020 under the EFOP-3.6.1-16-2016-00015.

Conflicts of Interest: The author declares no conflict of interest.

\section{References}

1. Hale, J.K.; Verduyn Lunel, S.M. Introduction to Functional Differential Equations; Springer: New York, NY, USA, 1993.

2. Győri, I.; Ladas, G. Oscillation Theory of Delay Differential Equations with Applications; Oxford University Press: New York, NY, USA, 1991.

3. Győri, I.; Krisztin, T. Oscillation results for linear autonomous partial delay differential equations. J. Math. Anal. Appl. 1993, 174, 204-217. [CrossRef]

4. Pituk, M. Asymptotic behavior and oscillation of functional differential equations. J. Math. Anal. Appl. 2006, 322, 1140-1158. [CrossRef]

5. Pituk, M. A note on the oscillation of linear time-invariant systems. Appl. Math. Lett. 2012, 25, 876-879.

6. Pituk, M. A Perron type theorem for positive solutions of functional differential equations. Electron. J. Qual. Theory Differ. Equ. 2018, 57, 1-11. [CrossRef]

7. Engel, K.-J.; Nagel, R. One-Parameter Semigroups for Linear Evolution Equations; Springer: New York, NY, USA, 2000.

8. Pazy, A. Semigroups of Linear Operators and Applications to Partial Differential Equations; Springer: New York, NY, USA, 1983.

9. Arino, O.; Győri, I. Necessary and sufficient condition for oscillation of a neutral differential system with several delays. J. Differ. Equ. 1989, 81, 98-105. [CrossRef]

10. Krisztin, T. Nonoscillation of functional differential equations of mixed type. J. Math. Anal. Appl. 2000, 245, 326-345. [CrossRef]

11. Henry, D. Small solutions of linear autonomous functional differential equations. J. Differ. Equ. 1970, 8, 494-501. [CrossRef]

12. Verduyn Lunel, S.M. A sharp version of Henry's theorem on small solutions. J. Differ. Equ. 1986, 62, $266-274$. [CrossRef]

13. Diekmann, O.; van Gils, S.A.; Verduyn Lunel, S.M.; Walther, H.-O. Delay Equations. Functional-, Complex-, and Nonlinear Analysis; Springer: New York, NY, USA, 1995.

14. Mallet Paret, J. Morse decomposition for delay-differential equations. J. Differ. Equ. 1988, 72, $270-315$. [CrossRef]

15. Cao, Y. The discrete Lyapunov function for differential delay equations. J. Differ. Equ. 1990, 87, 365-390. [CrossRef]

16. Arino, O. A note on "The discrete Lyapunov function ...". J. Differ. Equ. 1993, 104, 169-181. [CrossRef]

17. Cooke, K.L.; Verduyn Lunel, S.M. Distributional and small solutions for time-dependent linear delay differential equations. Differ. Integral Equ. 1993, 6, 1101-1117.

18. Mallet Paret, J.; Sell, G. The Poincaré-Bendixon theorem for monotone cyclic feedback systems with delay. J. Differ. Equ. 1996, 125, 441-489. [CrossRef]

19. Braverman, E.; Hasík K.; Ivanov, A.I.; Trofimchuk, S. A cyclic system with delay and its characteristic equation. Discrete Contin. Dyn. Syst. Ser. S 2020, 13, 1-29. [CrossRef]

20. Garab, Á. Absence of small solutions and existence of Morse decomposition for a cyclic system of delay differential equations. J. Differ. Equ. 2020, 269, 5463-5490. [CrossRef]

(C) 2020 by the author. Licensee MDPI, Basel, Switzerland. This article is an open access article distributed under the terms and conditions of the Creative Commons Attribution (CC BY) license (http://creativecommons.org/licenses/by/4.0/). 